\title{
COMPETENCIAS EN GERONTOLOGÍA: IMPERATIVO DEMOGRÁFICO
}

\section{Mabel T. López Ortiz ${ }^{1}$}

\section{Resumen}

Este artículo tiene como objetivo principal fundamentar el imperativo de desarrollar competencias en gerontología en los y las futuras trabajadoras sociales en Puerto Rico. Con este objetivo se discuten los hallazgos de un estudio exploratorio sobre las competencias en gerontología que poseen los y las estudiantes del curso electivo: La Gerontología y la Práctica del Trabajo Social del Programa de Bachillerato en Trabajo Social de la Universidad de Puerto Rico, Recinto de Río Piedras, año académico 2008-2009. Se incluyen datos importantes de los hallazgos de una investigación realizada por estudiantes de maestría de la Escuela Graduada de Trabajo Social, Beatriz Lasalle, sobre intereses y preferencias profesionales del estudiantado en nuestro bachillerato. El primer estudio resultó de una propuesta y proyecto auspiciado por el Council of Social Work Education (CSWE, por sus siglas en inglés) en su Instituto de Desarrollo Curricular del Gero-Ed Center. Las competencias en gerontología se definen como la demostración de las destrezas de intervención con la población de personas de edad avanzada, que se fundamentan en el conocimiento de esta etapa del ciclo de vida y en los valores de apreciación y respeto por la dignidad y derechos a la libre determinación, así como la participación en un contexto social de leyes y servicios inclusivos y equitativos de estas 
personas. En tiempos de precariedad en los servicios dirigidos a esta población, así como un aumento en la población de este sector se justifica la discusión del tema sobre las competencias que requieren los y las trabajadores sociales en Puerto Rico. Esta investigación fue autorizada por el Comité para la Protección de Seres Humanos en la Investigación del Recinto de Río Piedras, CIPSHI.

\section{Abstract}

This article have the principal objective of sustained the imperative of teach gerontological competences in our future social work professionals in Puerto Rico. With this purpose it discusses the findings of an exploratory study of gerontological competences in the students of an elective course: Gerontology and Social Work, Sub graduated Program of Social Work, University of Puerto Rico, Rio Piedras Campus, 2008-2009 academic year. Share also important findings of research conducted by graduate students of the Graduate School of Social Work, Beatriz Lasalle, on interests and career preferences of students of the Sub graduated Program. The first study was a proposal and project sponsored by the Council of Social Work Education (CSWE) in Curriculum Development Institute of the Gero-Ed Center. Gerontological competencies defined as demonstrating the skills of intervention with the population of elderly, which are based on knowledge of the life cycle stage and the values of appreciation and respect for the dignity and rights self-determination and participation in a social context of laws, and inclusive, equitable services to these people. In times of insecurity in the services for this population, as well as demographic increase, is necessarily the discussion of the issue of skills required by social workers in Puerto Rico. This research was approved by IRB Campus of Río Piedras, CIPSHI.

Introducción

¿Qué significan las competencias en gerontología para la profesión de trabajo social? Al presente, pueden significar el reto de enfrentar la realidad de una población que envejece y se proyecta para el 2012 con rostro femenino. Significan las madres, abuelas y mujeres que recibirán los servicios de las y los profesionales en trabajo social en un futuro cercano. No me adscribo solamente a Centros de Envejecientes, Edad Avanzada o de Oro, me refiero al porciento de personas que demográficamente existirán tanto nacional como mundialmente, por el aumento en la expectativa de vida, así como, por la realidad globalizada de este perfil humano que responde entre otros factores a los flujos migratorios y bajas tasas de natalidad (Hooyman, 2009; Sánchez, 2003). Esta situación se describe en Puerto Rico en el Informe Sobre el Perfil Demográfico, de la Oficina de la Procuradora de las Personas de Edad Avanzada, (OPPEA, 2007) con una proyección de hasta un $38 \%$ de personas de 60 años o más para el 2050. Además del hecho de que la mujer posee una expectativa de vida mayor que el hombre, nuestro país enfrenta un fenómeno adicional que aporta al envejecimiento de nuestra población con rostro femenino, y es la pérdida de nuestros jóvenes relacionada al trasiego de drogas, estilos de vida violentos y perfiles masculinos agresivos. En los últimos años se ha mostrado un patrón de homicidios en jóvenes varones entre las edades de 20 a 29 años transformando el perfil demográfico de nuestro país al descrito antes (Policía de Puerto Rico, 2009; Sánchez, 2008). En este panorama se entremezclan elementos de diversidad humana como el género y la cultura que invitan a la reflexión del tema y al el inicio de líneas de 
investigación que deben explorarse, y que a través de este artículo dejo a la reflexión e interés de las y los trabajadores y trabajadoras sociales, lectoras del mismo. Por estas razones el objetivo principal en este escrito es discutir el imperativo de desarrollar competencias en gerontología en los y las trabajadoras sociales de nuestro país.

Este objetivo demanda exponer los hallazgos de un estudio exploratorio sobre las competencias en gerontología o gerontológicas, que poseen los y las estudiantes del curso electivo TSOC 4195: La Gerontología y la Práctica del Trabajo Social del currículo del Programa de Bachillerato en Trabajo Social de la Universidad de Puerto Rico, Recinto de Río Piedras, y otro grupo de estudiantes que no pasan por la formación académica del mismo. También quiero incluir unos datos importantes de los hallazgos de una investigación realizada por estudiantes de maestría de la Escuela Graduada de Trabajo Social, Beatriz Lasalle, sobre intereses y preferencias profesionales del estudiantado en nuestro bachillerato. El primer estudio fue realizado por mi participación en un proyecto auspiciado por el Council of Social Work Education (CSWE, por sus siglas en inglés) en su Instituto de Desarrollo Curricular del Gero-Ed Center, el cual aprobó una propuesta de mi autoría presentada para la infusión curricular de competencias en nuestro Programa en el 2007. En este estudio apliqué una escala de valoración individual creada por esta instancia -la cual traduje al español con la autorización del Gero-Ed Center (véase Apéndice A)- al grupo de estudiantes de este curso electivo en el año académico 2008-2009, y a un grupo de estudiantes que no habían tenido la experiencia académica del curso electivo, con el objetivo de comparar ambas muestras. Esto fue una muestra por disponibilidad y el protocolo de investigación fue debidamente autorizado por el Comité para la Protección de Seres Humanos en la Investigación de nuestro Recinto, CIPSHI.

\section{Formación en Trabajo Social y Gerontología}

En la formación de la profesión en trabajo social, miramos y analizamos los graves problemas del maltrato infantil e intrafamiliar, y en ocasiones olvidamos el problema del maltrato a la persona envejecida tanto por su familia, como por la sociedad aportando a la invisibilidad del mismo. La dinámica familiar cuando tenemos personas de edad avanzada puede cambiar y trascender de la estabilidad a aspectos negativos relacionados con esta etapa de vida y que no deben distanciarse de la óptica del estudio de estas realidades (Glendenning, 2000). Esta población está vulnerable ante los cambios físicos, emocionales, económicos y sociales que enfrenta en esta etapa de su ciclo de vida. Usualmente las personas enfrentamos esta etapa en el ciclo de vida, con prejuicios y mitos construidos socialmente que se agravan ante el poco conocimiento e invisibilidad del tema, implicando complejidad y poca aceptación.

En Puerto Rico, a pesar de la existencia de la Ley 121 del 12 de julio de 1986, enmendada el 12 de agosto del 12 de agosto de 1995 , la cual protege a esta población del maltrato y violación de derechos, es poca la discusión y conocimiento de la misma. Inclusive la Oficina de la Procuraduría de las Personas de Edad Avanzada, OPPEA con sus funciones educar y fungir como sistema asesor y de apoyo social para nuestros adultos mayores es un organismo poco conocido. La situación y condición social actual de la población de personas de edad avanzada, nos dirige a la discusión del tema 
de la injerencia del trabajo social y el trabajo de apoyo con esta población. Existe un carácter de urgencia de ganar conocimientos y competencias en gerontología por varias razones algunas ya incluidas en mi introducción pero que intento abundar en el artículo, que pueden permitir lograr prevenir algunos de los problemas sociales relacionados con esta población, sin intentar que los mismos se perciban fragmentados de otras situaciones o cuestiones sociales. La OPPEA en su Informe Anual (2010), resume la situación y condición actual de esta población en los que sobresalen problemas precarios como una alta incidencia de contagio por VIH/SIDA de 1,514 casos reportados; maltrato de diferentes tipos perpetrados a esta población en un $16.5 \%$ de casos denunciados para el 2003, según un estudio citado en este informe del Recinto de Ciencias Médicas; un sector en aumento de abuelos y abuelas criando nietos y nietas; un registro de 377 casos de víctimas de violencia doméstica; por último el discrimen por edad en el trabajo con un registro de 172 personas.

Por una vía o nivel de intervención, debemos afirmar que ante la realidad y la falta de servicios para las personas de edad avanzada, así como la posibilidad de perder los existentes, tenemos la responsabilidad de enfrentar la política pública y como otro de nuestros escenarios de trabajo. Los problemas sociales y económicos que enfrentan nuestros y nuestras viejas en este panorama, laceran el compromiso de los y las trabajadoras sociales con el principio de justicia social, lo que requiere nuestra responsabilidad de prepararnos para atender y mirar a un sector invisible de nuestro país. De retos es nuestro camino, no sólo servimos niños y adolescentes tenemos una población muy valiosa en aumento, que espera y requiere del trabajo social en toda agencia y programa público y privado.
Según Sánchez (2006) se justifica el interés de la profesión de trabajo social en temas de gerontología ante los efectos demográficos globales, el impacto en la economía desde una mirada de la construcción de la pasividad de esta etapa y los efectos que políticamente esta población puede representar como un sector significativo en decisiones de política pública. Desde la década de 80 en nuestro país se discutía el "imperativo gerontológico" por varios grupos de estudios (Cáceres, 2003; Oliver, 2007; Sánchez, 2005). A tono con esta realidad los espacios académicos, en particular nuestras escuelas de formación en trabajo social públicas como privadas, deben estar en camino a conectarse con la idea de reafirmar el estudio y la infusión de las competencias en gerontología en sus programas. Ante este panorama los programas que forman al futuro y futura trabajadora social deben asumir el reto de integrar contenido y el desarrollo de competencias que nos permitan sensibilidad ante los y las participantes de edad avanzada. El CSWE, organización que acredita los programas de trabajo social subgraduados y graduados en Puerto Rico, ha comenzado a discutir la necesidad de un modelo de programa de estudio que se caracterice por integrar conocimientos, valores y destrezas gerontológicas en todos los cursos (Gero-Ed Center, 2009). Los modelos curriculares propuestos por este organismo, incluyen la especialización de algunos programas, la integración del tema en algunos cursos, o la infusión curricular la cual implica transformar los cursos medulares con el contenido de las competencias en gerontología (Hooyman, 2006). Específicamente se definen como competencias en gerontología, la demostración de las destrezas de intervención con la población de personas de edad avanzada que se fundamentan en el conocimiento de esta etapa del ciclo de vida y en los valores de apreciación y respeto 
por la dignidad y derechos a la libre determinación, así como la participación en un contexto social de leyes y servicios inclusivos y equitativos de estas personas (Damron-Rodríguez, et al., 2009). Las competencias en gerontología no tan solo deben enseñarse a nuestro estudiantado, si no que debemos buscar alternativas de medir e identificar la evidencia que nos muestre el desarrollo de las mismas. Estas competencias también deben alinearse según las guías de acreditación, con la misión y objetivos de los programas de formación, por lo cual se convierte en un proyecto académico completo en cualquier nivel académico de los programas que estén acreditados por el CSWE. (EPAS, 2008).

Específicamente, las competencias en gerontología deben responder al contexto social del país en referencia, cumpliendo entonces con el análisis incluido imperativo demográfico, las condiciones sociales en las que se encuentra esta población envejecida y otros múltiples factores en una era de globalización de la producción, tecnología y el conocimiento (Damron-Rodríguez, Volland, Wright \& Hooyman, 2009).

\section{Competencias en Gerontología: Compromiso Ético y Político}

El concepto de competencias no es uno nuevo en la literatura académica, para el CSWE (2008) resulta ser un imperativo y requisito en los programas académicos en todos los niveles que esta entidad acredita. En términos generales cuando hablamos de competencias, describimos las prácticas medibles compuestas por los conocimientos, valores y destrezas. Estas competencias deben incluirse en los currículos académicos y alinearse a las diez competencias definidas por la política educativa de los estándares de acreditación de este cuerpo (Gero-Ed Center, 2009). Las mismas deben ser ajustadas a todo tipo de población, nivel de intervención o áreas temáticas de nuestra formación y práctica profesional. Atendiendo a estas guías y a la necesidad de medir las competencias en el estudiantado, el Gero-Ed Center construyó su escala de competencias gerontológicas basadas en las diez competencias generales sobre conocimientos, valores y destrezas que se requieren en la intervención del trabajo social con personas de edad avanzada (véase Apéndice A). Para este cuerpo acreditador y los programas especializados en el tema de gerontología, el desarrollo de competencias gerontológicas en el futuro profesional en trabajo social, queda justificado.

Pero en el ámbito de la realidad social de nuestra práctica con personas de edad avanzada, ¿qué significan las competencias geriátricas o gerontológicas? Debemos comenzar con la reflexión crítica sobre las actitudes y aprecio que deberían mostrar los y las trabajadoras sociales en la intervención con personas viejas. Escoger una población por interés, preferencia o predilección puede guiar le selección de nuestros escenarios de trabajo. La discusión en este artículo puntualiza hacia el momento de enfrentarnos a una realidad que incluye a personas de edad avanzada en cualquiera de los escenarios de trabajo, ya que no se adscribe a nuestros intereses, se refiere a datos y proyecciones reales. Este contexto traduce la aplicación de las competencias en gerontología al aprecio de esta población desprovista de servicios en la actualidad y que se proyecta a un aumento considerable en condiciones desfavorables.

Quiero incluir en la discusión y justificación del desarrollo de competencias en gerontología y el tema de la diversidad humana, no tan solo en el estudiantado en formación en trabajo social, si no

VOCES DESDE EL TRABAJO SOCIAL NÚM.1 
también el sector de profesionales en todos los escenarios públicos y privados. La diversidad humana es un tema muy discutido actualmente que incluye en su definición conceptual y operacional, al sector de personas ancianas y los problemas de discrimen por edad, así como la vulnerabilidad de esta población en desventaja (Oliver, 2007). Estas situaciones competen a la profesión de trabajo social, ya que como conocemos esta es una fundamenta de valores de justicia social y defensa de los derechos humanos, así como del compromiso con las poblaciones vulnerables como lo son nuestros viejos y viejas.

A la diversidad de características en los seres humanos que son nuestro compromiso, se suma el factor de la edad y ciclo de vida de esta población, mostrando así la complejidad de las múltiples identidades e intersecciones de características humanas. Esta intersección de identidades, recrudece la vulnerabilidad a ser víctima de opresión por los sistemas sociales formales e informales de las personas de edad avanzada. Varios autores y autoras, discuten como otro punto de tensión y que tiene implicaciones para la práctica del trabajo social con esta población, precisamente el aumento en el perfil diverso de las personas de edad avanzada (Hooyman, 2009; Orel, 2004; Sánchez, 2003). El aumento en población de mujeres viejas, pobres, de personas viejas gays, lesbianas y bisexuales, de personas migradas viejos y viejas -sin documentos por ejemploimplican situaciones de suma vulnerabilidad por el discrimen histórico de los diferentes ejes o características de estas identidades. Características diversas como el género, orientación sexual, clase o condición social, viudez, cultura o etnia entre otras, recrudecen el panorama que he intentado describir de falta de servicios de apoyo para atender las necesidades y situaciones sociales del sector de personas de edad avanzada.

Esta discusión es requerida ante el propósito de desarrollar una visión integrada e inclusiva, valores y ética en la persona que ejerce el trabajo social como su profesión. Debemos reconocer que la diversidad humana, en particular las diferencias de edad entre los seres humanos, también requieren de la búsqueda y autoevaluación de prejuicios, mitos y estigmas construidos socialmente que nos pueden alejar de la defensa de una práctica sostenida por competencias gerontológicas requerida en nuestros tiempos. Esta mirada sobre la realidad de un perfil que envejece, la multiplicidad de realidades y condiciones sociales de vulnerabilidad, nos guía a la reflexión y necesidad de integrar competencias de intervención dirigidas a atender a las personas envejecientes.

Antes de alcanzar la meta de que las y los trabajadores sociales posean conocimientos, una base de valores y competencias que aseguren una práctica sensible, inclusiva y asertiva con el propósito de fortalecer a las personas de edad avanzada, debemos lograr que todas y todos nos sintonicemos con la realidad del envejecimiento poblacional y la valoración de esta población como los y las futuras y principales participantes a asistir en nuestra intervención.

\section{Competencias en Gerontología: Una mirada Crítica}

Aunque nos enfrentamos al reto de analizar de forma crítica las implicaciones del discurso sobre las competencias requeridas bajo la política, economía y necesidades sociales de un sistema capitalista, surgen otras preguntas como, ¿dónde quedan los y las profesionales en trabajo social ya formados que ofrecen servicios directos y que

VOCES DESDE EL TRABAJO SOCIAL

NÚM.1 
trabajan con esta población a través de individuos, familias, grupos y comunidades donde se encuentran las personas de edad avanzada? Estas y estos trabajadores sociales poseen una experiencia práctica que desde sus historias podrá dar cuenta del significado de los cambios que han enfrentado los programas de servicios donde nos movemos como profesionales, pero exige otros conocimientos, destrezas y valores dirigidos a atender a este sector. Es mi interés alertar a todas las personas que ejercen el trabajo social en Puerto Rico, sobre el aumento de una población que requiere de servicios dirigidos a necesidades particulares, que traen consigo esta etapa del ciclo de vida y la falta de estos servicios por las nuevas políticas públicas establecidas en Puerto Rico. Un ejemplo es el intento en la política pública de eliminar la Oficina de la Procuraduría de las Personas de Edad Avanzada, la cual encontró resistencia por el personal de esta oficina, como por el sector organizado de viejos y viejas en nuestro país, logrando así la permanencia de este servicio.

Las necesidades de esta población se posicionan ante las demandas de una sociedad capitalista de producción de bienes y servicios que relega la persona adulta que envejece ante principios de "darle espacio a la juventud", y "no apreciar y valorar" lo que la persona de edad avanzada puede aportar y ofrecer con sus recursos a nuestra sociedad. La promoción político-partidista que observamos a través de los medios televisivos, en la pasada campaña electoral para las elecciones en el 2008, contaba con la estrategia de llamar la atención de esta población, ofreciendo erradicar la soledad y la marginación; estrategia que puede impresionarnos como una muy acorde con la realidad demográfica que exponemos. Esta estrategia utilizada por las personas en carreras políticas en el país al momento de reclutar votos, no debe ser olvidada por nosotros y nosotras las trabajadoras sociales, ya que puede ser utilizada por estos sectores para exigir sus derechos (Juntos hacia el Cambio, 2009-2010).

Cuando la prioridad de las personas que dirigen y crean la política pública del país no es proteger los derechos humanos que poseen las personas de edad avanzada, se violenta y oprime de forma institucionalizada a esta población. Los derechos básicos de salud, vivienda y seguridad para este sector deben ser la prioridad del sistema de apoyo social en el país, pero se encuentran en condición de precariedad según los informes de la OPPEA, 2007. El rol del trabajo social como profesión en este aspecto nos reitera la función política de defensa de derechos humanos de esta población, en adición a la lucha por la política social y servicios que necesita la población de edad avanzada. A partir de toda esta discusión y el fundamento de la necesidad de reafirmar nuestro compromiso con las personas de edad avanzada, así como el compromiso que como académica demanda este panorama, surgió el interés de acercarme al estudiantado para explorar una perfil de estos con relación al tema de las competencias en gerontología, así como conocer si el curso electivo de Gerontología podía convertirse en el primer paso en el aprendizaje de conocimientos básicos, destrezas y valores a desarrollarse para una práctica futura con estas personas.

\section{Resumen de Hallazgos}

Deseo discutir un resumen de los hallazgos de este estudio para sumar a la justificación de alguna manera, tanto de la necesidad de comenzar a discutir el futuro sobre el perfil de los y las participantes que atenderemos en la diversidad de escenarios de práctica, y también la importancia de tener cursos en formación académica, como en 
la educación continuada de los y las profesionales del trabajo social. Específicamente, el estudio sobre las competencias en el estudiantado del programa subgraduado de Trabajo Social, fue uno exploratorio con enfoque cuantitativo donde se administró el cuestionario de Evaluación de Competencias en Gerontología (ver Apéndice A) a una muestra de un grupo de 10 estudiantes del curso electivo de Gerontología, y otro grupo de 17 estudiantes que no habían tomado el curso con el propósito de comparar las competencias que ambos poseían, en el año académico 2008-2009. Los objetivos principales eran medir la autoevaluación de competencias en ambos grupos y ofrecer recomendaciones para la infusión de las competencias en gerontología en los contenidos de los cursos del currículo de Programa Subgraduado de Trabajo Social en la Universidad de Puerto Rico, Recinto de Río Piedras.

La escala utilizada fue creada y desarrollada por el Programa de Práctica de la Fundación John A. Hartford, y luego revisada y adaptada por el Centro de Gero-Ed del CSWE. Esta escala la traduje con la colaboración de la asistente de investigación, Aydilmarie La Croix, con un doble cotejo y por último una contratraducción de la Profesora Magaly Ruíz, consultora del Departamento de Trabajo Social para el 2008. La escala es una autoevaluación de cuatro áreas: valores, ética y perspectivas teóricas: Conocimientos y base valorativa, la cual se aplica a través de las Competencias, Ponderación, Intervención y Servicios, Programas y Políticas para Personas de Edad Avanzada.

Para describir la escala, la valoración comenzaba en 0 hasta 4, donde cero tenía el significado de: No hábil o competente y el cuatro significaba: Experta o experto en habilidad. La muestra encuestada se posicionaba autoevaluando su percepción de las competencias adquiridas y que se describía en esta escala. Los criterios de participación del primer grupo de la muestra fueron estar matriculados en el curso electivo TSOC 4195, y ser estudiantes del Programa de Trabajo Social. El segundo grupo como requisito debía ser estudiante del Programa de Trabajo Social y no podían haber tomado el curso electivo en Gerontología. En general la muestra de estudiantes que poseían el curso electivo TSOC 4195 mostraron una autoevaluación de sus competencias en los valores de la escala de habilidad y competencia avanzada a expertos en la competencia o sea de 4-5. Por otro lado la muestra de estudiantes que no habían tomado el curso mostró percibirse con debilidades en las competencias que apuntan a una autoevaluación de carecer de destrezas y competencias gerontológicas o sea de $0-3$. Estos hallazgos pueden apuntar a confirmar la importancia del curso electivo, TSOC 4195 en el desarrollo de competencias en gerontología y la meta de graduar estudiantes con competencias en la intervención con personas de edad avanzada que según el CSWE, deben poseer los programas de formación en Trabajo Social. Debo reconocer como limitación la cantidad del estudiantado que participó. Esta cantidad se debe a que otra parte del grupo de esta electiva eran estudiantes de otros programas por lo cual tuvieron que ser excluidos y excluidas.

Por otro lado, la investigación realizada por Avilés y Rodríguez (2009), con el propósito de identificar las ideologías, preferencias profesionales, y actitudes hacia el concepto de género de un grupo de estudiantes del Programa Subgraduado de Trabajo

VOCES DESDE EL TRABAJO SOCIAL

NÚM.1 
$\overline{\text { Social en su último año de formación, encontró falta de interés y }}$ preferencia en intervenir con viejos/as, por el estudiantado del Programa Subgraduado en Trabajo Social. La muestra fue de 67 participantes (10\% masculinos y $90 \%$ féminas) de los cuales 13 eran estudiantes del Recinto de Humacao y los restantes 54 del Recinto de Río Piedras. Esta muestra priorizó las poblaciones de mayor interés comenzando con adolescentes (72.3\%), adultos jóvenes $(70.8 \%)$, niños y niñas $(69.3 \%)$, adultos $(70.8 \%)$ y por último, viejos y viejas (47.7 \%), (Avilés \& Rodríguez, 2009, p. 87). En esta misma investigación se compararon las muestras del estudiantado de Puerto Rico con un grupo de Israel, encontrando que en ambos países hay menor predilección hacia trabajar con la población de edad avanzada (Weiss, 2005).

Estos datos nos enfrentan a la contradicción entre el contexto real del imperativo demográfico con el interés y la preferencia de los y las estudiantes en proceso de formación en Trabajo Social. Estos y estas trabajadoras sociales serán quienes se integrarán a la política social y servicios que se ofrecen a nuestros individuos y familias puertorriqueñas encontrándose con una gran cantidad de participantes de edad avanzada. El ideal o imaginario de la práctica con otras poblaciones resulta no ser de forma exclusiva del estudiantado de trabajo social puertorriqueño, ya que parece ser el resultado de ideologías y política neoliberal que excluye inclusive de las expectativas profesionales en el ejercicio de la práctica del trabajo social, atender a la población de viejos y viejas.

\section{Múltiples Realidades: Reflexiones sobre el Trabajo Social en Gerontología}

Las actitudes creadas a través de la socialización en el

VOCES DESDE EL TRABAJO SOCIAL actual contexto social, político y económico, se distancian de las caras de nuestras poblaciones que envejecen, el equipaje individual se adiciona al interés que mostramos por el aprecio y trabajo con las personas de edad avanzada. Este contexto es participe de esta distancia en la intervención con personas de edad avanzada no tan sólo a nivel micro sino también hasta el macro; la política pública dirigida e esta población puede carecer de criterios que se atemperen a la realidad social de nuestras y nuestros viejos y viejas, pero las instituciones sociales informales como los medios de comunicación, han aportado a los prejuicios y estereotipos de productividad, belleza, aislamiento y estigma que ha caracterizado la descripción de la vejez.

Frente a este contexto y el imperativo de formar trabajadores sociales con competencias gerontológicas, queda la decisión personal como salida a la intervención del y la profesional del Trabajo Social. Según Goncalves (2009) la literatura apunta a tres niveles personales que llevan al estudiantado a seleccionar inclusive un curso en gerontología, como lo son el contacto previo con adultos de edad avanzada, -ya sea en un escenario informal o formalmenteel género y la edad. Nuestros y nuestras estudiantes, así como los y las trabajadores sociales en servicio, han guiado su interés a la práctica con personas de edad avanzada por intereses personales, lo cual no necesariamente ha surgido de su formación académica.

El aumento en la población de edad avanzada es parte de nuestra realidad, la necesidad de trabajar a todos los niveles desde la construcción de nuestro proyecto profesional tanto ético como político, para prevenir las consecuencias que pueda traer este perfil demográfico, es la acción próxima a seguir. El estudiantado ha mostrado cuál es su interés, y contradice el futuro demográfico que

VOCES DESDE EL TRABAJO SOCIAL

NÚM.1 
proyectamos. Estos hallazgos me llevan a sugerir problematizar esta contradicción, y atender el imperativo demográfico desde todas sus dimensiones y escenarios. La academia tiene un reto que cumplir. Son los programas de formación los que deben iniciar esta acción, incluyendo en sus propuestas curriculares ya sean modelos especializados, de integración o de infusión del tema y contenido de la población de edad avanzada considerando no tan sólo las competencias sugeridas por los organismos acreditadores, si no incluyendo la discusión de los significados y múltiples realidades de los currículos y la población de edad avanzada en nuestro país y realidad puertorriqueña. No debemos excluir la aportación en la investigación social, la cual se torna en un deber con líneas de investigación interesantes e innovadoras como las identidades de género, orientación sexual, y perfil laboral de nuestras viejas y viejos. Los organismos profesionales como el Colegio de Profesionales de Trabajo Social, poseen las estructuras para aportar al conocimiento sobre temas relacionados con esta población, de los y las que ya formados y formadas, se encuentran ofreciendo servicios desde diferentes escenarios. La acción en otros escenarios como son las comunidades, grupos y la política social son retos que contextualizan este problema y a los que no debemos descartar como espacios de reflexión crítica y acción en defensa de la justicia social y derechos humanos de nuestras personas de edad avanzada.

Problematizar nuestra realidad, los y las viejas son parte de nuestro futuro, la ausencia de actitudes de valor y aprecio a estos debe retarse para comenzar a atender el imperativo demográfico, las condiciones de precariedad social-derechos humanos, y la exclusión social.

\section{Referencias}

Avilés, Raúl \& Rodríguez, Rafael (2009). Trabajo Social en el Contexto Internacional. Tesis de Maestría: Escuela Graduada de Trabajo Social, UPR.

Beamer, Marion \& Miller, Don.A. (1998). La práctica clínica del trabajo social con personas mayores. España: Paidós.

Cáceres, Virgen (2003). "Diálogo Comunitario: Personas de Edad Avanzada, Voces que se Sienten": Identificación de Necesidades, Problemas y Recursos. Universidad de Puerto Rico: CIS

Council on Social Work Education. (2008). Èducational policy and accreditation standards. Tomado de http://www.cswe.org/ File.aspx $? \mathrm{id}=13780$

Damrom-Rodríguez, Joan,Volland, Patricia, Wright, M. Elizabeth \& Hooyman, Nancy (2009). Competency-Based Education. En Hooyman, N. (Ed). Transforming Social Work Education. (pp.21-49). VA: Council of Social Work Education.

Gero-Ed Center. (2009). Advanced Gero: Social Work Practice. VA: Council of Social Work Education.

VOCES DESDE EL TRABAJO SOCIAL 
Glendenning, Frank (2000). ¿Qué entendemos por negligencia y maltrato a los ancianos? En El maltrato a las personas mayores (pp. 17-56). Barcelona: Paidós.

Goncalves, Daniela C. (2009). From loving Grandma to working with olders adults: Promoting Positive Attitudes Towards Aging. Educational Gerontology, 35, 3, p. 202-235.

Hooyman, Nancy (Ed.) (2009). Tansforming Social Work Education. VA: Council of Social Work Education.

Hooyman, Nancy (2006). Achieving curricular and organizational change. VA: Council of Social Work Education.

Oficina de la Procuradora de las Personas de Edad Avanzada. (2007). Perfil demográfico de la población de 60 años o más en Puerto Rico. PR:Autor.

Oficina de la Procuradora de las Personas de Edad Avanzada. (2010). Informe Anual 2010. Recuperado en http://www. ogave.gobierno.pr/

Oliver, Marlén (2007). Diversidad y vejez: mitos y realidades. En Rosalie Rosa (Ed.), La Diversidad Cultural: Reflexión crítica desde un acercamiento interdisciplinario. San Juan: Publicaciones Puertorriqueñas.

VOCES DESDE EL TRABAJO SOCIAL $\overline{\text { Orel, Nancy A. (2004). Gay, lesbian and bisexual elders. Journal of }}$ Gerontological Social Work, 43:2, 57-77.

Partido Nuevo Progresista. (2009-2010). Juntos hacia el Cambio. Recuperado de http://www.fortunogobernador.com/images/ PLANDEGOBIERNOPNP2009-2012.pdf

Policía de Puerto Rico. (2009). Víctimas de Homicidio por Edad y Género. Estado Libre Asociado: Autor. Recuperado en http://www.gobierno.pr/PoliciaPR/Estadisticas/

Sánchez, Carmen (Ed). (2003). La mujer de Edad Mayor en una Sociedad Feminizada. I Seminario Centroamericano y El Caribe Red de Estudios de la Vejez. Costa Rica: EUNED.

Sánchez, Carmen (2005). Familia y persona de edad mayor. (Ed.) II seminario de Centroamérica y el Caribe Red de estudios de la vejez, ATLANTEA.

Sánchez, Carmen (1999). Gerontología Social. Puerto Rico: Publicaciones Puertorriqueñas.

Sánchez, José (2008, 22 de octubre). El país pierde la sangre joven. El Nuevo Día, p.12.

Ley \# 121, Para establecer la Política Pública y la Carta de Derechos de las Personas de Edad Avanzada (12 de Julio de 1986). PR: Estado Libre Asociado.

VOCES DESDE EL TRABAJO SOCIAL

NÚM.1 
Markson, Elizabeth (2003). Social gerontology today: an introduction. California: Roxbury Publishing Company.

Weiss, Idith (2005). Interest in working with the elderly: A cross-national study of graduating social work students. Journal of Gerontological Social Work, 41(3), 379-392.

\section{Apéndice A: Escab de Cotnpetencios en Gerantologí}

\section{CSWE COUNCIL ON SOcial Work Education}

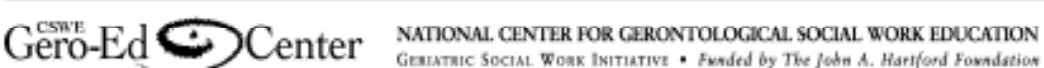
I.

\section{Escala de Competencias Geriátricas en elia Estudiante de Trabajo} Social'

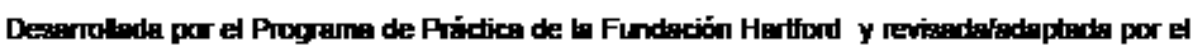
Centro de Gero-Ed det CSWE

\section{Para Utilizarse en la Valoración de las Competencias Acanzadas por} elina Estudiante

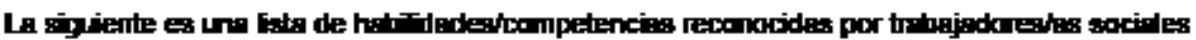

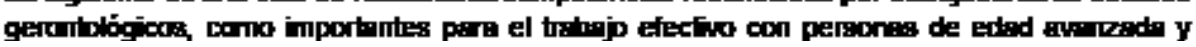

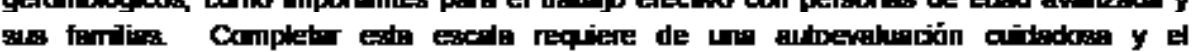

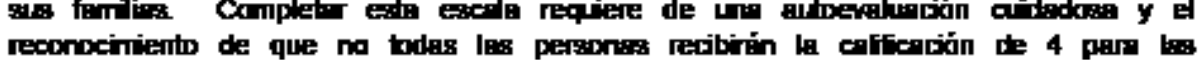

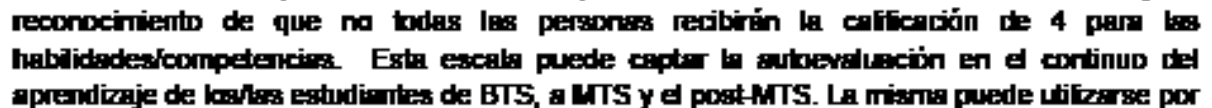

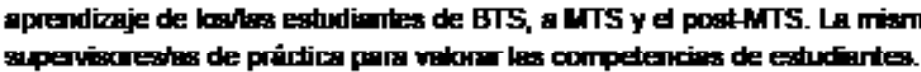

\section{metrucciones:}

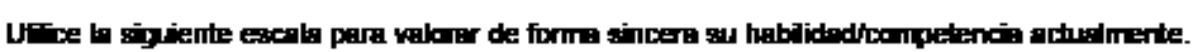

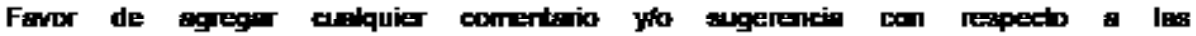

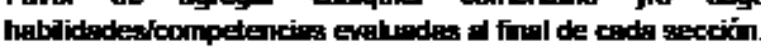

Vako de la Escolns

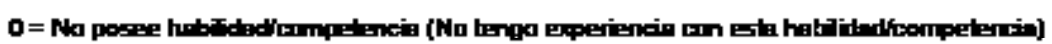

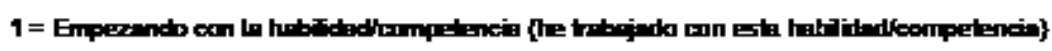

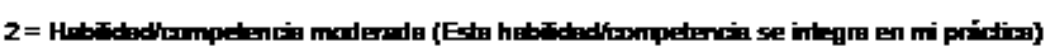

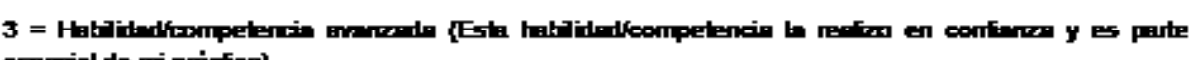
exrivil de mi pripicics\}

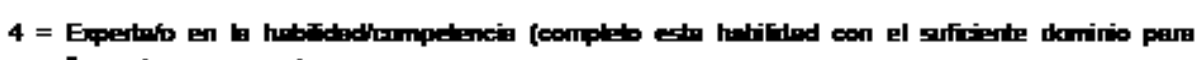
enseir a ches pessmas]

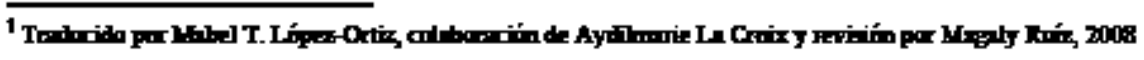




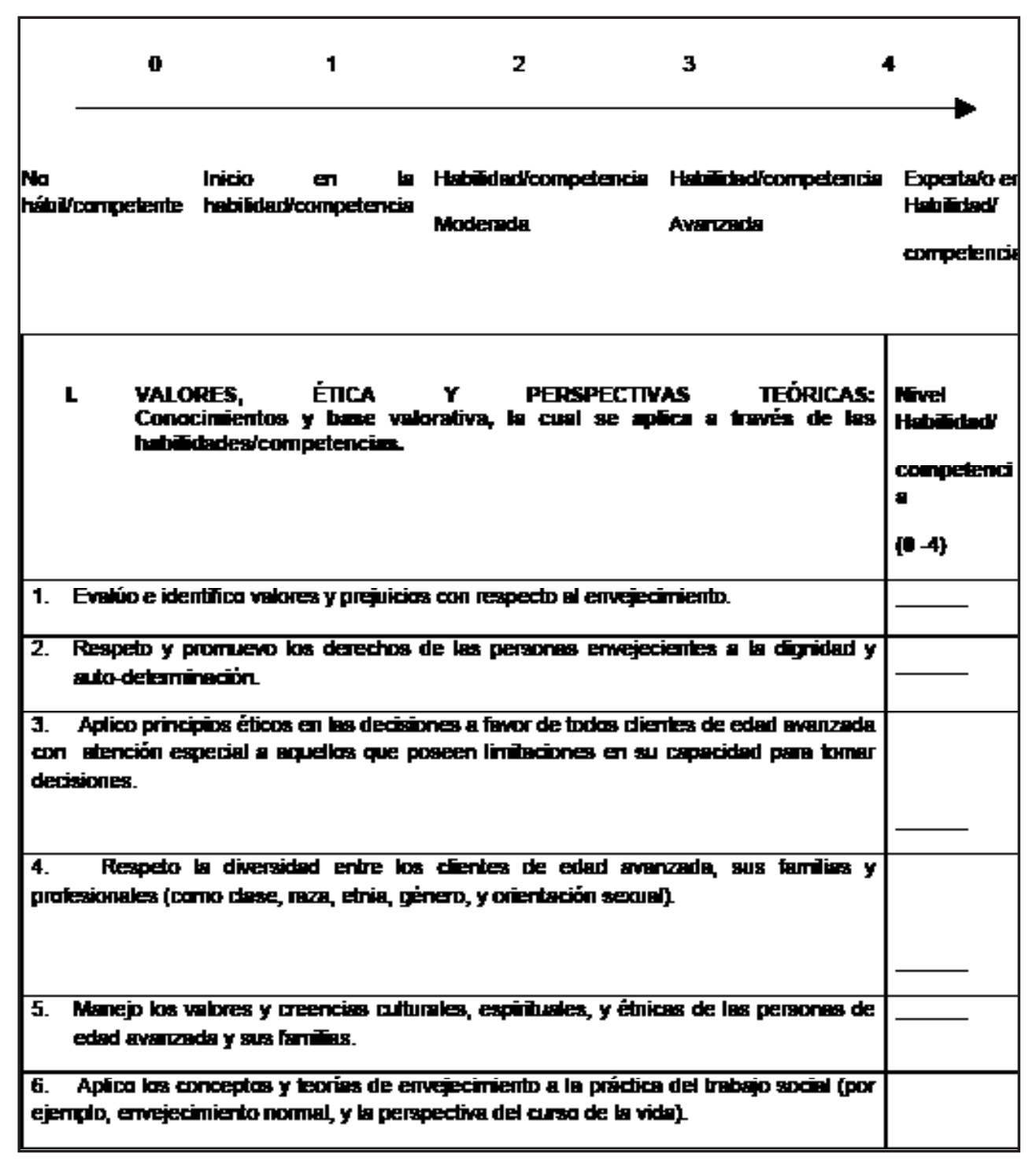

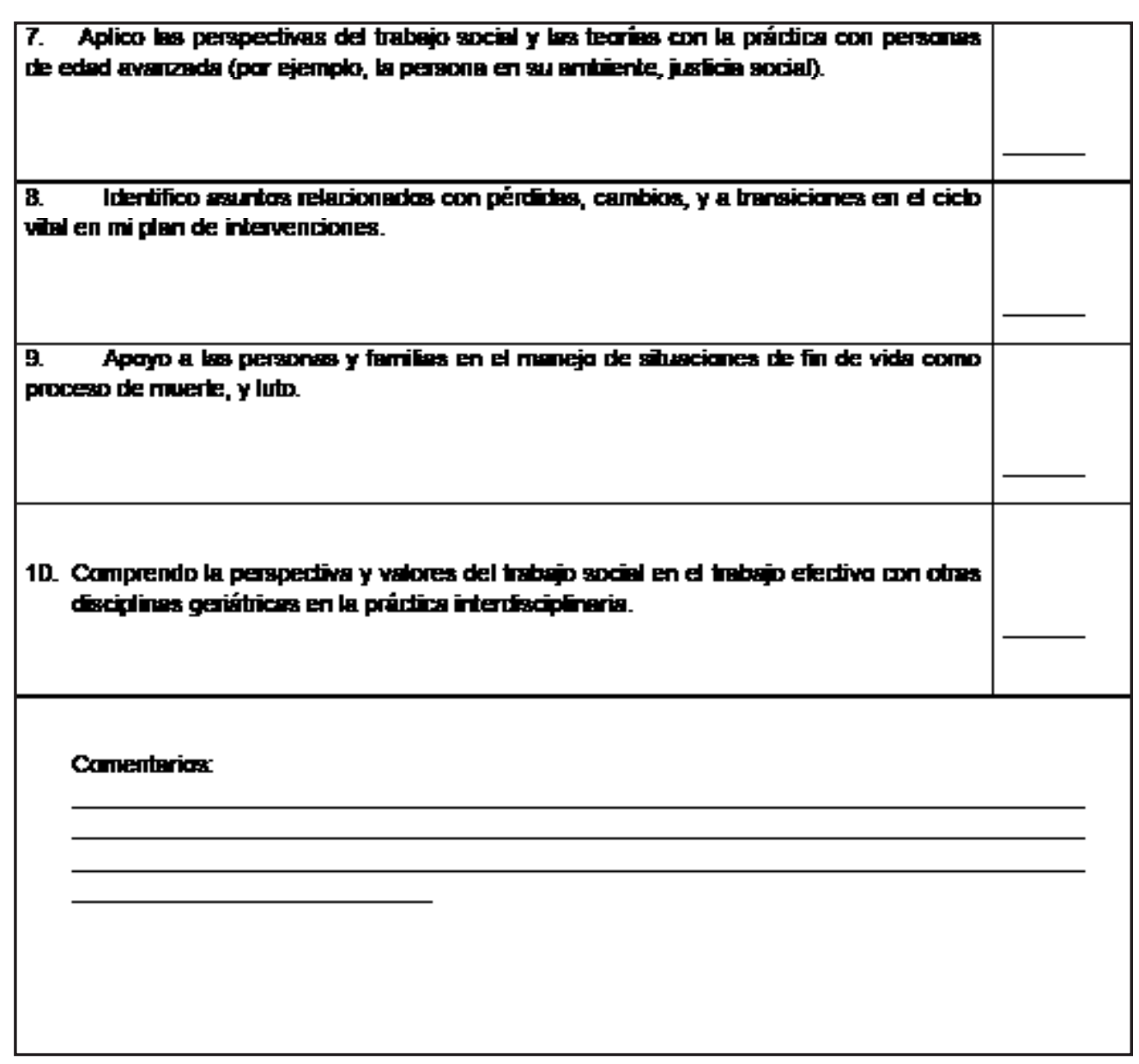




\begin{tabular}{|c|c|}
\hline II. PONDERACKN: & 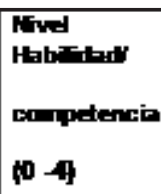 \\
\hline \multicolumn{2}{|l|}{ 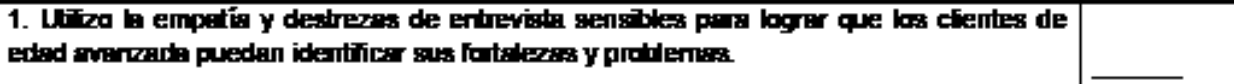 } \\
\hline 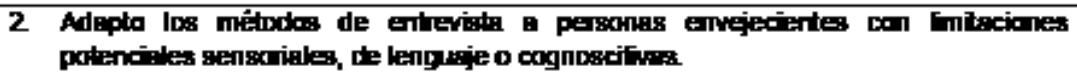 & \\
\hline \multicolumn{2}{|l|}{ 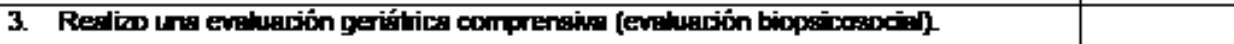 } \\
\hline \multicolumn{2}{|l|}{ 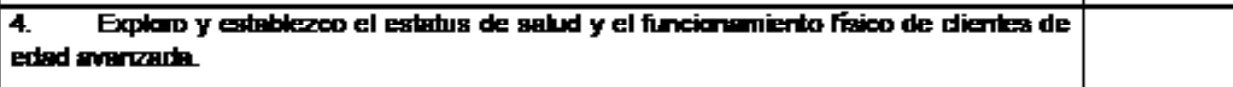 } \\
\hline 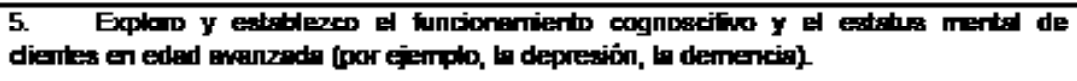 & \\
\hline \multicolumn{2}{|l|}{ 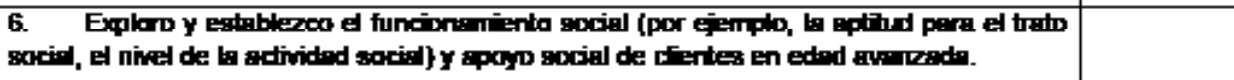 } \\
\hline \multicolumn{2}{|l|}{ 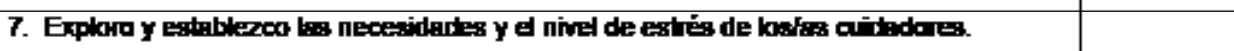 } \\
\hline 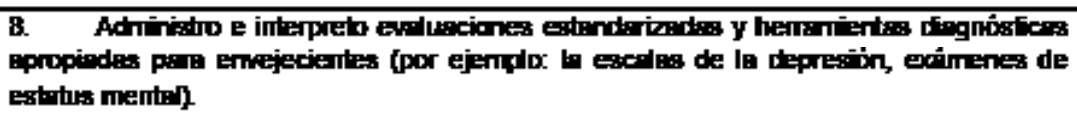 & \\
\hline
\end{tabular}

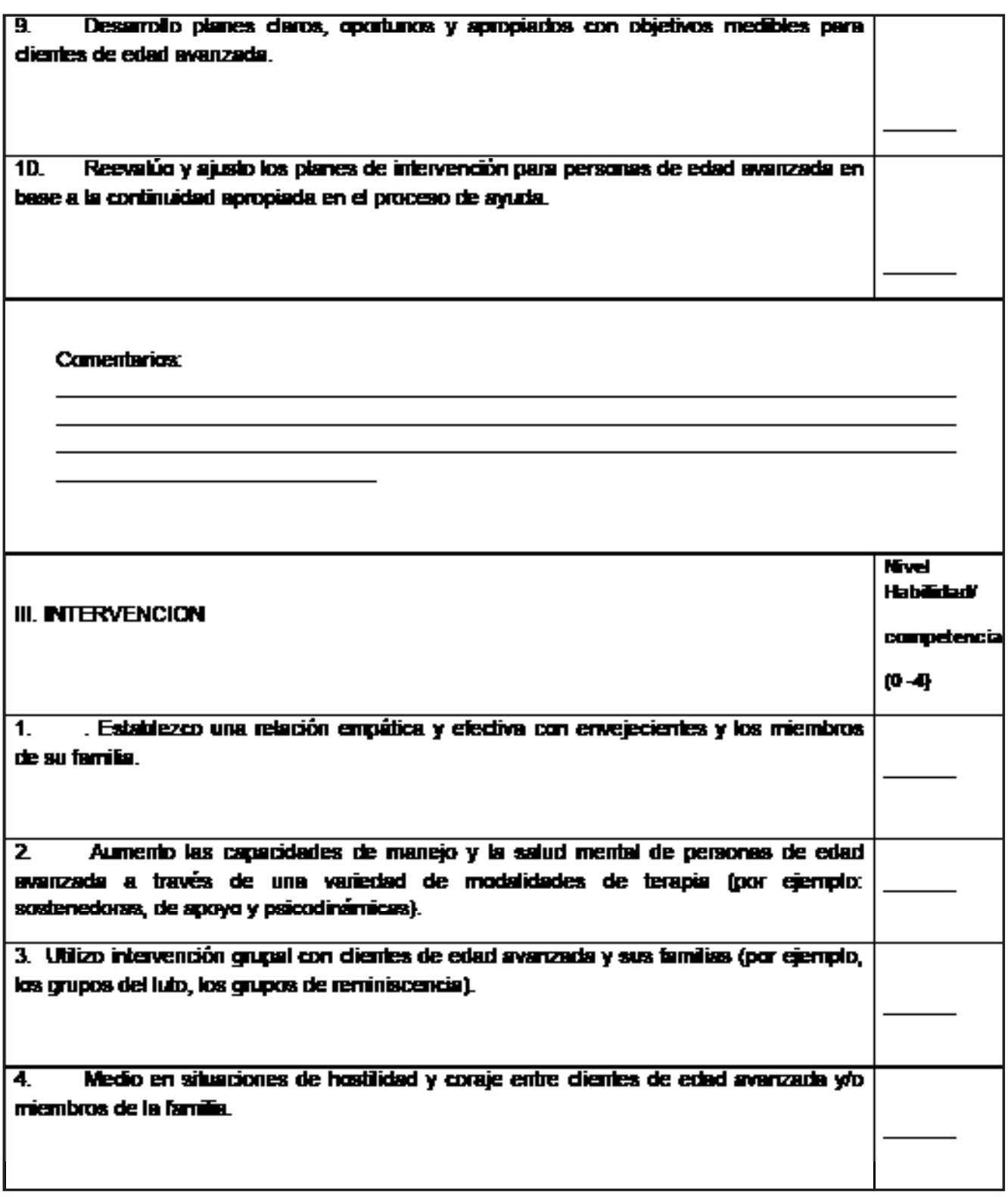




\begin{tabular}{|c|c|}
\hline 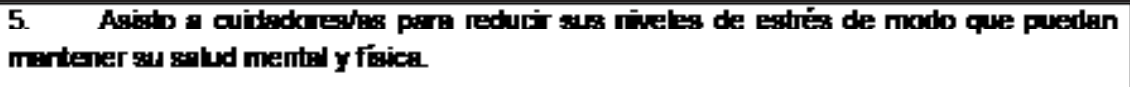 & \\
\hline 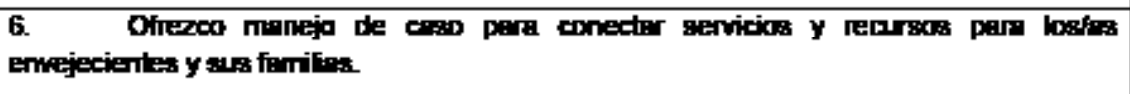 & \\
\hline 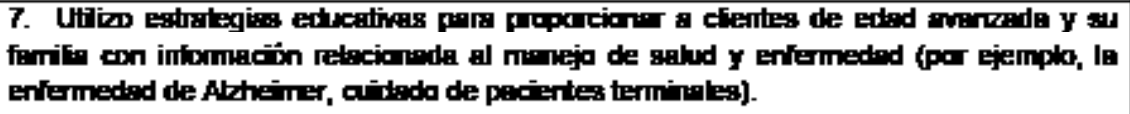 & \\
\hline 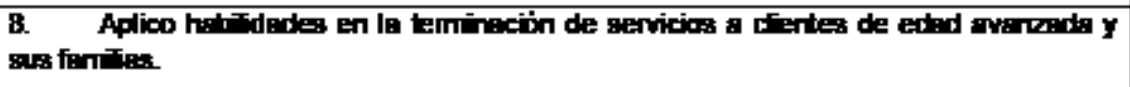 & \\
\hline 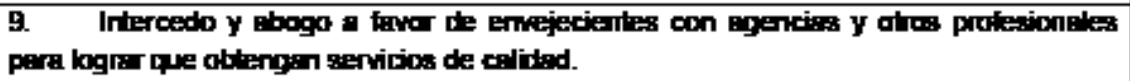 & \\
\hline 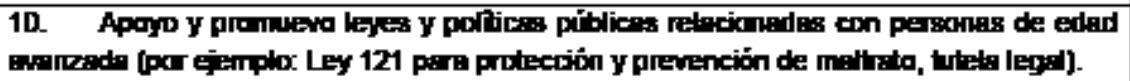 & \\
\hline Cementeriox & \\
\hline
\end{tabular}

\begin{tabular}{|c|c|}
\hline 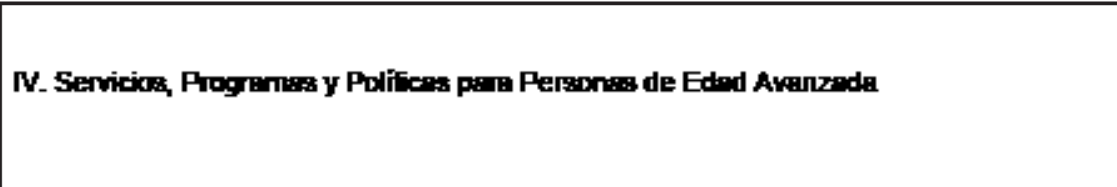 & 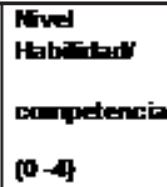 \\
\hline 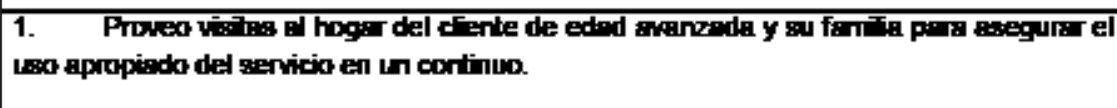 & \\
\hline 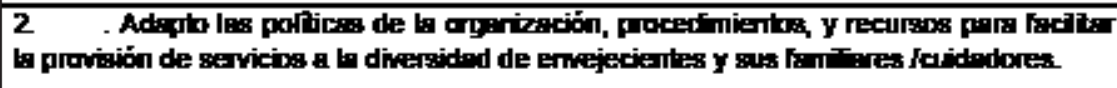 & \\
\hline 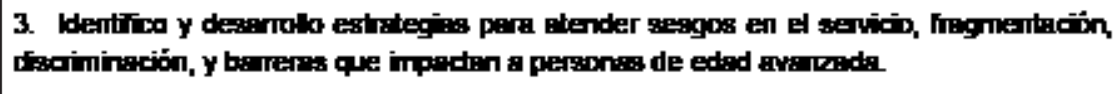 & \\
\hline 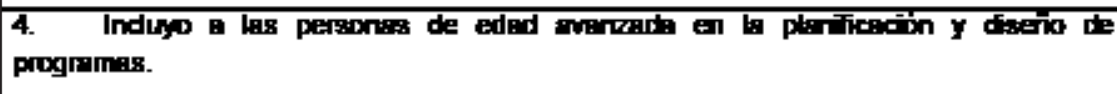 & \\
\hline 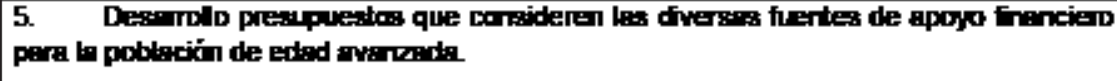 & \\
\hline 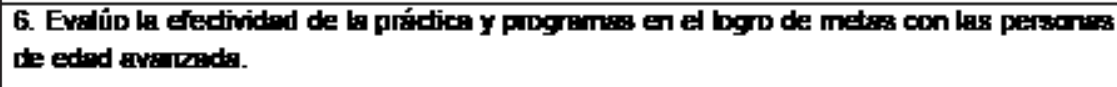 & \\
\hline 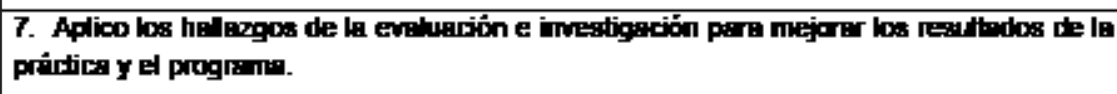 & \\
\hline 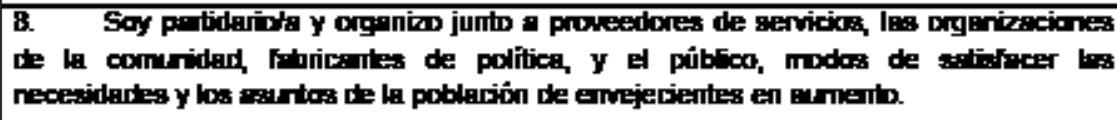 & \\
\hline
\end{tabular}




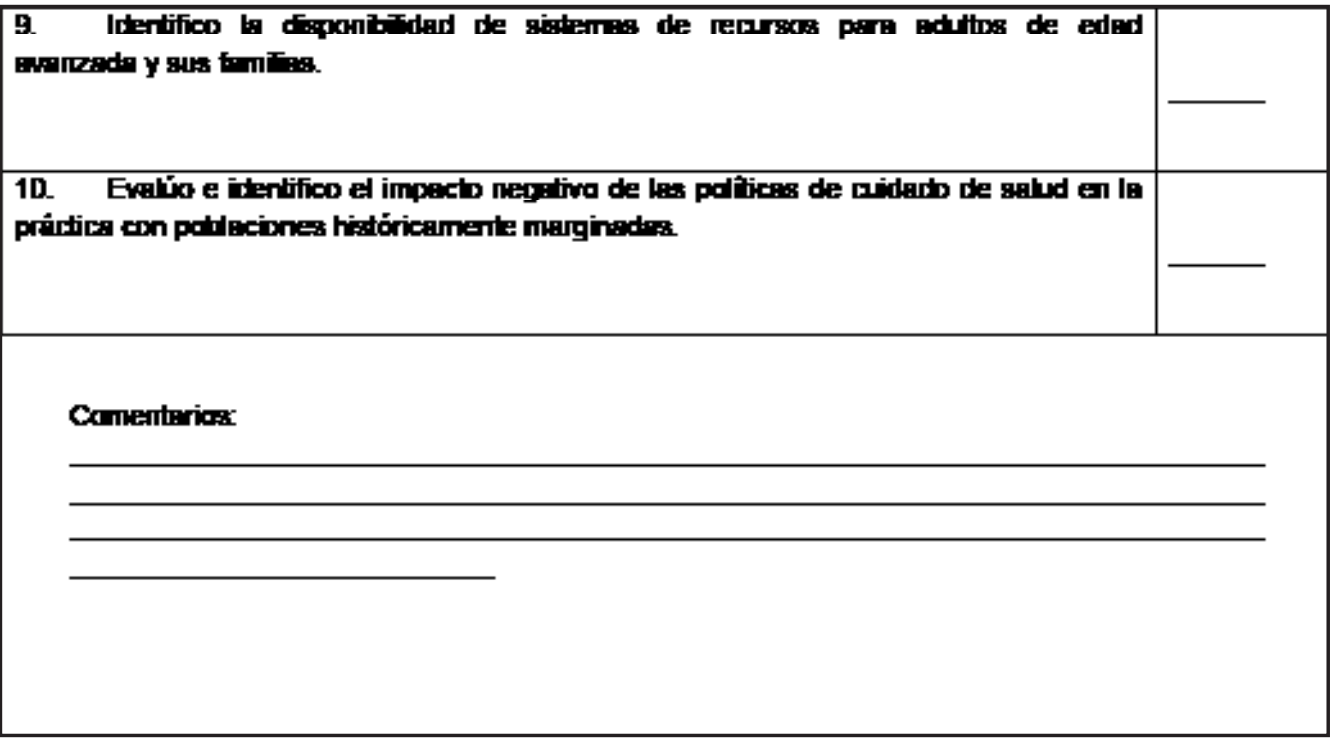

